\title{
Computing Capacity and Connectivity in Cognitive Radio Ad-Hoc Networks
}

\author{
Qiang-Sheng $\mathrm{Hua}^{\dagger}$, Haisheng Tan ${ }^{\dagger}$, Yuexuan Wang ${ }^{\dagger}$, Hongxing $\mathrm{Li}^{\ddagger}$ \\ Dongxiao $\mathrm{Yu}^{\ddagger}$, Francis C.M. Lau ${ }^{\ddagger}$, Chuan $\mathrm{Wu}^{\ddagger}$ \\ ${ }^{\dagger}$ Institute for Theoretical Computer Science, Institute for Interdisciplinary Information Sciences, \\ Tsinghua University, Beijing, P.R. China \\ $¥$ Department of Computer Science, The University of Hong Kong, Pokfulam Road, Hong Kong, P.R. China
}

\begin{abstract}
We present some unique challenges in cognitive radio ad-hoc networks (CRAHNs) that are not present in conventional single-channel or multi-channel wireless ad-hoc networks. We first briefly survey these challenges and their potential impact on the design of efficient algorithms for several fundamental problems in CRAHNs. Then, we describe our recent contributions to the capacity maximization problem [29] and the connectivity problem [32]. The capacity maximization problem is to maximize the overall throughput utility among multiple unicast sessions; the connectivity problem is to find a connected subgraph from the given cognitive radio network where each secondary node is equipped with multiple radios. By assuming the physical interference model and asynchronous communications, we reformulate the above two problems where the capacity maximization problem is to find the maximum number of simultaneously transmitting links in secondary networks, and the connectivity problem is to construct a spanning tree over secondary networks using the fewest timeslots. We discuss the challenging issues for designing distributed approximation algorithms and give a preliminary framework for solving these two problems.
\end{abstract}

\section{INTRODUCTION}

With the ever increasing demand for wireless services, due partly to the explosive growth of multimedia transmissions, the wireless spectrum has become a scarce resource. Cognitive Radio is a promising technology that can alleviate the spectrum scarcity problem in wireless communication. It allows the unlicensed secondary users to utilize the temporarily unused licensed spectrums, referred to as white spaces, without interfering with the licensed primary users. Cognitive Radio Networks (CRNs) are believed to be the next generation of communication networks and the field has attracted many researchers in both academia and industry recently [48]. In this paper, we consider Cognitive Radio Networks without a centralized authority, which are called Cognitive Radio AdHoc Networks (CRAHNs) [1].

Different from conventional wireless ad-hoc networks where each node has a fixed set of channels, in CRAHNs, through spectrum sensing and spectrum database querying, each secondary user (SU) can measure the currently available channels, i.e., channels not used by the primary users (PUs). Due to the behaviors of the PUs and the possible mobility of the secondary users (SUs), the available channels of the SUs have the following characteristics [6]:

- Spatial Variation: SUs at different positions may have different available channels;
- Spectrum Fragmentation: the channels available to a SU may not be continuous;

- Temporal Variation: the available channels of a SU may change over time.

By these characteristics, even the most fundamental operations in traditional wireless ad-hoc networks will become quite challenging to realize in CRAHNs. In the following, we briefly survey how some of these unique challenges may impact basic protocol design for the Rendezvous problem, the neighbor discovery problem, and the broadcasting problem in CRAHNs.

\section{Protocol Design Challenges in CRAHNs}

The first challenge CRAHNs may face is about message delivery. In traditional wireless ad-hoc networks, since both the sender and the receiver can share the same channel or set of channels, sending a message is easy as long as the receiver is in the sender's transmission range. However, to successfully send a message transmission in a CRAHN, besides the transmission range requirement, we also need to ensure the sender and receiver will quickly find a common channel. The act of quickly finding a common channel is called Rendezvous [14], [45] or Discovery [5]. The two-user Rendezvous problem can be defined as follows. Consider a pair of secondary users, Alice and Bob. One of them knows his/her local available channels, but is unaware of the other's. In each time slot, Alice and Bob will select a local available channel and try to connect with each other. A rendezvous occurs or a discovery is successful if Alice and Bob both choose the same channel at the same time. The objective is to derive channel selection strategies for Alice and Bob such that they can achieve discovery using the minimum number of attempts. Since we do not assume common control channels in CRAHNs, the Rendezvous problem is clearly a challenge especially in multiple-user cases with asynchronous communications.

For synchronous communications, Jump-Stay [34] is the state-of-the-art distributed algorithm that provides guaranteed blind rendezvous (without a common control channel) in CRAHNs. In this method, users jump on the available channels in the jump-pattern while they stay on a specific channel in the stay-pattern. The authors prove that the jump-stay method has bounded Maximum Time-to-Rendezvous (MTTR) for both the two-user and multiple-user cases. However, the expected 
Time-to-Rendezvous (E(TTR)) could be exponentially large for the multiple-user case. For asynchronous communications with only two users, the authors in [5] proposed a distributed rendezvous algorithm with guaranteed expected discovery time. The authors also show that this expected discovery time is optimal under the assumption of infinitely many channels. For asynchronous communications with multiple users, to our best knowledge, there exist no distributed approximation algorithms. Interested readers may refer to [33] for a survey on various rendezvous algorithms for both two-user and multipleuser scenarios.

Another challenging problem is neighbor discovery [3], [26]. Neighbor discovery in traditional wireless ad-hoc networks can be realized by efficient local broadcasting algorithms [52], [55]. However, in CRAHNs, a SU's neighbor is determined not only by its transmission range but also by the fact that if they have a common channel. Thus in order to find all neighbors, each SU needs to implement local broadcasting on all of its available channels. This can get quite complicated when intertwining with the Rendezvous problem and the interference issue. In [26], by assuming synchronous communications, the authors give a brute-force neighbor discovery algorithm time complexity $O(m n)$ where $m$ is the number of available channels that the CRAHNs can use and $n$ is the number of secondary nodes, labeled from 1 to $n$. In [3], [4], by assuming asynchronous communications, the authors give distributed algorithms for both single-hop and multi-hop cognitive radio networks. For single-hop networks, the neighbor discovery task is mainly carried out by the computed leader. However, for the multi-hop scenario, the author assumes the presence of a leader in the network before performing neighbor discovery. Thus this algorithm does not work for CRAHNs since there are no leaders. In addition, both of these two works did not take interference into account.

The third protocol design challenge faced by CRAHNs is broadcasting. Deriving a minimum-latency broadcasting in traditional wireless ad-hoc networks is an NP-hard problem. In CRAHNs, it is even harder since each node may have a different set of channels. We need to first determine the sender's broadcast channel sequence (the sequence of channels to broadcast on) and the receivers' listening channel sequence. To reduce the broadcasting delay, the sequence should include as few channels as possible. For example, in [25], the key technique is to let each SU only broadcast on the minimum set of channels (Essential Channel Set, abbreviated ECS). The ECS is a subset of the SU's available channels and it is computed to cover all the SU's geographic neighbors. A similar idea for reducing the broadcast delay is also introduced in [41], where each SU needs only to broadcast on a downsized channel set which is a subset of its available channels. This downsized channel set is calculated based on both the PUs' and the SUs' distributions and it must ensure that each sender has at least one common channel with each of its neighbors. In [2], [4], the authors introduce the minimal time broadcasting problem which is to determine the minimum broadcast schedule length for a CRAHN. The authors formulate this problem as an
Integer Linear Programming (ILP) problem and present two heuristics.

Other than fundamental operations such as the above, researchers are also interested in relatively more complex operations in CRAHNs. Next we describe our own work on two such operations, from which we can see that efficient protocols at different network layers need to be jointly considered, such as channel (spectrum) assignment, link scheduling, routing and rate control. These two operations are capacity maximization [29] which is to maximize the throughput utility of end-to-end unicast sessions, and connectivity which is to find a connected subgraph from a given cognitive radio network [32].

\section{CAPACITY MAXIMIZATION FOR END-TO-END UNICAST SESSIONS}

In cognitive radio networks, the primary user occupation pattern is mostly dynamic which complicates the optimization (e.g., capacity maximization) of data dissemination among secondary users. When multiple data dissemination sessions (among secondary users) co-exist, a fundamental challenge is how to judiciously allocate the spectrum and schedule the transmissions such that the available spectrum is fully exploited to achieve the maximum network-wide throughput utility. This requires a cross-layer design, for optimal transport, network and MAC layer decisions to be made. At the transport layer, source nodes properly adjust data injection rates for lower layers to handle; at the network layer, a relay node strategically decides the next-hop relay for each data session to be forwarded to; at the MAC layer, the available spectrum is carefully and dynamically allocated for transmission between pairs of nodes. All of these have to adapt to the volatile channel occupancy patterns of the primary users while trying to maximize the end-to-end throughput utility among all data sessions.

Another dimension of complexity is added if we drop the usual assumption of fully collaborative or completely selfish ${ }^{1}$ data relay among secondary users and explicitly model the social selfishness of the users. In real-world networks, e.g., civilian networks [22], [28], users have social ties at various strength levels. Naturally, a user would prefer helping others with whom there is a strong social tie, and less so for nodes with weak social ties. Such social selfishness of users has brought new challenges to the design of efficient data dissemination protocols, especially when making routing decisions and link capacity allocation [31]. For example, a node with high link capacity and low hop count to the destination, which although appears favorable, may not constitute a good relay option if it is not willing to assist in the data session. Therefore, traditional routing protocols, i.e., those based on

\footnotetext{
${ }^{1}$ For complete selfishness, the literature has focused on incentive design, e.g., [23], [49], [57], which is an orthogonal topic to our work. In real-world, the social ties tend to be fixed and stable. Thus, we design our protocol with social selfishness being a user demand, instead of designing incentive mechanisms to entice the users to collaborate.
} 
link capacity or hop count, is no longer suitable in a socially selfish network.

In our work, we consider social selfishness of secondary users, and design a joint end-to-end rate control, routing, and channel allocation protocol that can maximize the overall throughput utility among multiple unicast sessions. We are aware of only one paper, by Li et al. [31], which has the same assumption of social selfishness as ours. They investigate routing design in socially selfish delay tolerant networks, where the probabilities that a node may forward traffics received from other nodes are differentiated. Unlike their work, we study a joint rate control, routing, and channel allocation scheme in a cognitive radio network, where we address social selfishness of users in their transmission scheduling of packets belonging to different by-passing data sessions according to the social ties between these users and the source/destination of each session.

Our design is rooted in Lyapunov optimization theory [37], where utility maximization and network stability are achieved by back-pressure scheduling of transmissions among packet queues at the network nodes. A salient contribution of our Lyapunov optimization is that finite buffer sizes are employed at each node with no-buffer-overflow guarantee.

Since the seminal work of Tassiulas et al. [44], backpressure protocols for maximum-weight scheduling, which schedule links with the largest product of link capacity and differential queue backlog, have been widely applied for utility maximization in multi-hop wireless networks [9], [10], [13], [46], [51]. It has been shown that optimal throughput can be achieved. However, most solutions are based on infinite node buffers, which is obviously an idealized and impractical assumption.

Venkataramanan et al. [46] suggested a way to minimize the cumulative buffer utilization along the path of a unicast flow so as to reduce the end-to-end delay of the flow. However, there is no finite-size guarantee for each buffer at the relay nodes. The challenge of using finite buffer in a back-pressure paradigm was not addressed until recently by Le et al. [27] and Neely [38]. Le et al. [27] have investigated optimal control of a wireless network with finite buffer for each by-passing session per relay node, but an infinite buffer is still necessary at each source node in the worst cases. In [27], the current queue size at each source node needs to be broadcast to all relay nodes, which incurs a high communication overhead. Our protocol avoids such a broadcast overhead. Neely [38] recently proposed an opportunistic scheduling protocol with bounded buffer size at each node for each data session, which simply drops the packets when a buffer becomes full. In [38], the throughput utility is only compared with that of a $T$-slot lookahead policy which is an offline policy with perfect knowledge of up to $T$ slots into the future. No analysis however is given on how close the throughput utility can approach optimality. To the contrary, we demonstrate with rigorous proof that a finite buffer size without the possibility of buffer overflow suffices at each node using our protocol which can achieve global throughput utility maximization.
Ding et al. [9], [10] have designed back-pressure protocols for routing with collaborative spectrum sensing, but without utility-optimality guarantee. Feng et al. [13] introduce a backpressure routing protocol with primal-dual decomposition. No analysis of buffer size is given. Xue et al. [51] propose a back-pressure throughput maximization protocol, under the constraints of bounded collision rates between secondary and primary users. The worst-case upper bound of buffer size at each node is derived, but the protocol cannot ensure that there will be no buffer overflow in situations where the buffer sizes are smaller than the upper bound. Our protocol provides that guarantee.

The contributions of our work can be summarized as follows:

- We model social selfishness of users by differentiated buffer sizes and relay rates allocated to data sessions of different source/destination pairs in a Lyapunov optimization framework for achieving throughput utility maximization in a cognitive radio network. To the best of our knowledge, this is the first work investigating the impact of social selfishness on protocol design in cognitive ratio networks.

- We propose a back-pressure-style joint end-to-end rate control, routing, and channel allocation protocol for optimal multi-session unicast data dissemination, and give a distributed implementation for it. More specifically, the rate control decision is made at each source node only based on its local queue lengths while each node determines the joint routing and channel allocation based on its own queue lengths and channel availabilities as well as those of its interfering nodes.

- First time in the literature of back-pressure protocols, our protocol requires only a finite buffer at each source or relay node with no buffer overflow, and is guaranteed to achieve an overall throughput utility that can be arbitrarily close to the ultimate optimum obtained when there is no constraint on buffer sizes.

- We demonstrate network stability and utility optimality of our protocol with rigorous theoretical analysis. Impact of social selfishness on throughput utility and end-to-end dissemination delay of different data sessions are further investigated using both case studies and empirical studies. An interesting discovery is that, contrary to the intuition that larger buffers should be provisioned to preferred data sessions, allocating smaller buffers to these sessions at nodes along their paths can actually lead to smaller endto-end delay, without sacrificing throughput.

\section{The Complexity of Connectivity}

In this section, we consider the connectivity problem of multiple links through channel assignment. In CRAHNs, connection between two nodes is not only determined by their distance and their transmission powers, but also by whether the two nodes have chosen a common channel. Due to the spectrum dynamics, communication in CRAHNs is more difficult than in the traditional multi-channel ad-hoc networks. 
Authors of [35], [39], [40], [50] studied the impact of different parameters on connectivity in large-scale CRAHNs, such as the number of channels, the density of primary and secondary users, the activity of PUs, the number of neighbors of SUs and the transmission power.

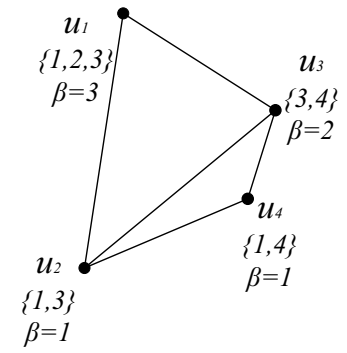

(a)

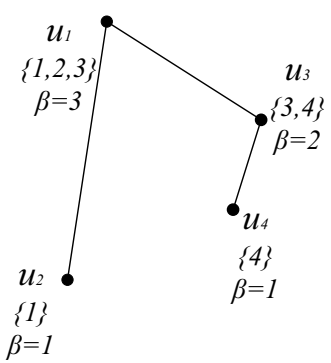

(b)
Fig. 1. a) The potential graph: the set besides each SU is its available channels, and $\beta$ its number of antennae. Two nodes do not have an edge (e.g., $u_{1}$ and $u_{4}$ ) because their distance exceeds at least one of their transmission ranges. b) The realization graph which is connected: the set besides each SU is the channels assigned to it.

We study the complexity of connectivity through channel assignment in a centralized fashion, i.e., each node knows the network topology, its neighbor's available channels and the number of antennae. We assume each SU $u$ is equipped with a number of antennas (radios), denoted as antenna budget $\beta(u)$, which is the maximum number of channels that $u$ can open (work on) simultaneously. A channel assignment is a way of opening the channels such that each SU opens at most $\beta$ channels and can only open its local open channels (the SU's available channels). We model the network as a potential graph $\mathcal{P G}=(E, V)$ and a realized graph before and after spectrum assignment respectively, where $V$ is the set of SUs. An edge $\{u, v\}$ in the potential graph means the two nodes can communicate as long as they choose a common available channel. Given a channel assignment, an edge in the potential graph is realized if the two nodes are indeed allocated a common channel. The realized graph under a channel assignment is a graph $\mathcal{R G}=\left(U, E^{\prime}\right)$, where $E^{\prime}$ is the set of realized edges in $E$. An example is shown in Fig. 1.

As shown in Fig. 1, SUs may be equipped with different numbers of antennae and the potential graph can be arbitrary. We study the special case when all the SUs have the same antenna budget. If all the SUs are homogenous and have a large enough transmission range, the potential graph will be a complete graph. For some hierarchically organized networks, e.g., a set of SUs being connected to an access point, the potential graph can be a tree. We also study these special cases. Exact algorithms are derived to determine connectivity for different cases. We list our results below [32].

- When the potential graph is a general graph, we prove that the problem is NP-complete even if there are only two channels through a reduction from the Uniform SAT problem. This result is sharp as the problem is polynomial-time solvable when there is only one channel. We also design exact algorithms for the problem. For the special case when all SUs have the same number of antennae, we prove that the problem is NP-complete when $k>\beta \geq 2$, where $k$ and $\beta$ are the total number of channels in the white spaces and the number of antennae in an SU respectively.

- When the potential graph is a complete graph $^{2}$, the problem is shown to be NP-complete even if each node can open at most two channels based on a reduction from the Hamiltonian Path problem. However, in contrast to the general graph case, the problem is shown to be polynomial-time solvable if the number of channels is fixed. In fact, we prove a stronger result saying that the problem is fixed parameter tractable when parameterized by the number of channels.

- When the potential graph is a tree, we prove that the problem is NP-complete even if the tree has depth one through a reduction from the vertex cover problem. Similar to the complete graph case, we show that the problem is fixed parameter tractable when parameterized by the number of channels.

Remark 1: To the best of our knowledge, this is the first work that systematically studies the algorithmic complexity of connectivity in CRAHNs with multiple antennas. However, our work does not address how to design distributed approximation algorithms for joint channel assignment and link scheduling for achieving network connectivity, especially under the physical interference model. In Section VI, we reformulate the connectivity problem for constructing a spanning tree in CRAHNs.

\section{COMPUTING THE CAPACITY IN CRAHNS}

In Section III, we have introduced our recent work on maximizing the throughput utility of end-to-end unicast sessions. There, we assumed the existence of a common control channel, the graph-based interference model and synchronous communications. In addition, our proposed distributed algorithm does not have a performance guarantee. In this section, we reformulate the capacity maximization problem without the above limitations. We first present our system model and the communication model which will also be used for the problem of computing the connectivity in the upcoming Section VI.

\section{A. System Model}

There are $M$ primary users, denoted by $S_{1}, S_{2}, \ldots, S_{M}$, and $N$ secondary users, denoted by $s_{1}, s_{2}, \ldots, s_{N}$; they are all placed on the plane based on some probability distribution, such as even distribution. There are $M$ orthogonal channels that form the universe channel set $\left\{c_{1}, \ldots, c_{M}\right\}$ and each primary user could randomly select a channel to transmit. All the primary and secondary users will use fixed transmission powers $P_{p}$ and $P_{s}$, respectively. The set of available channels for each secondary user $s_{i}$ is a subset of the universe channel set and the user $s_{i}$

\footnotetext{
${ }^{2}$ The complete graph is a special case of disk graphs.
} 
is unaware of the other secondary user $s_{j}$ 's available channels. Both the primary and the secondary nodes have only one radio meaning they can only operate on a single channel. We assume half-duplex mode and no common control channels. Finally, there is no global clock, and we assume asynchronous communications meaning that each secondary user may start the algorithm at anytime.

\section{B. Communication Model}

We assume the Signal-to-Interference-plus-Noise-Ratio model which is also called the physical model since it can reflect the physical reality somewhat accurately [55]. Different from graph-based interference models, the physical model takes the cumulative interference from all the other simultaneously transmitting links into account. If a secondary user $s_{i}$ would like to send a message to another secondary user $s_{j}$ on their common channel $c_{k}$, we say that the receiver $s_{j}$ successfully received the message from $s_{i}$ iff

$$
\frac{\frac{P_{s}}{d\left(s_{i}, s_{j}\right)^{\alpha}}}{\sigma^{2}+\sum_{s_{k} \neq s_{i}} a_{s_{k}}^{c_{k}} \frac{P_{s}}{d\left(s_{k}, s_{j}\right)^{\alpha}}+\sum_{S_{k}} a_{S_{k}}^{c_{k}} \frac{P_{p}}{d\left(S_{k}, s_{j}\right)^{\alpha}}} \geq \beta_{s} .
$$

Similarly, if a primary user $S_{i}$ would like to send a message to another primary user $S_{j}$ on their common channel $c_{k}$, we say that the receiver $S_{j}$ successfully received the message from $S_{i}$ iff

$$
\frac{\frac{P_{p}}{d\left(S_{i}, S_{j}\right)^{\alpha}}}{\sigma^{2}+\sum_{S_{k} \neq S_{i}} a_{S_{k}}^{c_{k}} \frac{P_{p}}{d\left(S_{k}, S_{j}\right)^{\alpha}}+\sum_{s_{k}} a_{s_{k}}^{c_{k}} \frac{P_{s}}{d\left(s_{k}, S_{j}\right)^{\alpha}}} \geq \beta_{p} .
$$

In these two inequalities, $d($,$) is the Euclidean distance$ function, $\sigma^{2}$ is the background noise variance and $a_{s_{k}}^{c_{k}} \in$ $\{0,1\}=1$ if the secondary user $s_{k}$ transmits on the channel $c_{k}, 2<\alpha<=6$ is the path loss exponent and $\beta_{s}\left(\beta_{p}\right)$ is the threshold value for the secondary user (primary user). Note that although the physical interference model does not consider the shadowing and multi-path fading effects of wireless transmissions, the authors in [11] prove that applying existing algorithms for the physical interference model in the Rayleigh-fading scenario loses only a factor of $O\left(\log ^{*} n\right)$ in the approximation guarantee. Thus, this observation supports the use of the physical interference model without considering the fading effects of wireless transmissions.

\section{Problem Definition}

We now reformulate the capacity maximization problem given the above system and communication models. Instead of maximizing the throughput utility of the end-to-end unicast sessions, we aim to maximize the number of simultaneously transmitting links over the secondary networks (assuming each link's rate is 1). To achieve this goal, we need to design a distributed approximation algorithm that jointly considers channel assignment and link scheduling. We need to emphasize that, from the SINR inequalities 1 and 2, we have to consider the cumulative interference from both secondary and primary users as long as they are operating on the same channel. Moreover, the SINR inequality 2 must be guaranteed first in the sense that they are primary users whose transmissions should not be influenced by secondary transmissions ${ }^{3}$.

\section{Related Work on Capacity Maximization}

The capacity maximization problem and its variations have been extensively studied in both conventional single-channel wireless ad-hoc networks [15], [24], [47] and cognitive radio ad-hoc networks [12], [16], [43]. In traditional single-channel wireless ad-hoc networks, by using uniform power assignment, i.e., all the users employ the same transmission power, the authors in [15], [47] have given constant factor approximation algorithms for the capacity maximization problem. If power control is allowed, there is also a constant factor approximation algorithm for the same problem by using the iterative power assignment. However, all these algorithms are centralized algorithms and these results can not be applied in CRAHNs. In order to maximize the total channel utilization for all secondary users, the authors in [20] proposed the maximum channel selection (MCS) problem and formulated it as a binary integer nonlinear optimization. The authors then proposed both centralized and distributed algorithms. However, they provided no worst-case performance guarantees. In addition, the paper considers synchronous communications. By employing the SINR model, the authors in [8], [56] studied the spectrum distribution problem which is to maximize the total number assigned channels for all secondary users. In the capacity maximization problem, we aim to maximize the maximum number of simultaneously transmitting links (only allowing a single assigned channel for each link); thus it is different from the spectrum distribution problem. In [16], the authors aim to find a maximum set of links that can be simultaneously scheduled without affecting a given set of previously assigned links. The paper gives a constant-factor centralized approximation algorithm under uniform power assignment. However, there is only one channel in the network and thus their algorithm cannot be applied in general CRAHNs where each secondary user may have a different set of available channels. Note that in order to maximize the capacity in general CRAHNs, channel assignment must be jointly considered with link scheduling. Note that, if there is only one channel in the network, although it obviates the channel assignment process, it loses another degree of freedom to further increase the network capacity. Assuming the protocol interference model, the authors in [43] present a family of heuristic algorithms for the capacity maximization problem, the max-min fairness problem and the proportional fairness problem. A closely related work to our capacity maximization problem just defined is that presented in [12]. By adopting the SINR model, the authors propose a heuristic algorithm without a worst case performance guarantee. In addition, this paper considers synchronous communications.

\footnotetext{
${ }^{3}$ We treat the secondary network as an overlaid network in which the primary user and secondary user could be operating on the same channel. However, if we consider the "spectrum hole" model [42] where the primary and secondary users will not operate on the same channel, the problem will become much easier since we only need to consider the SINR constraints in secondary networks.
} 


\section{E. Preliminary Framework}

Here we give a preliminary framework on how to schedule the maximum number of links in the same timeslot. According to the system model, the first step is to identify which links can be scheduled, i.e., to decide if there is a common available channel between two secondary users. This can be realized by performing a distributed neighbor discovery algorithm [3], [4]. Since there are no common control channels, the neighbor discovery algorithm entails an efficient two-user rendezvous. In addition, since the system does not have a global clock, only asynchronous neighbor discovery algorithms may be used. In order to deal with the asynchronous communication issues, by adopting the physical interference model, we have developed a family of distributed algorithms for the multiple-message broadcasting problem [53], the coloring problem [54] and the data aggregation problem [19] in single-channel wireless adhoc networks. A key technique used is to first elect the leaders and then let the leaders coordinate the wireless transmissions. A possible way for electing the leaders is to compute a maximal independent set over the secondary networks.

After performing neighbor discovery, each secondary user will know who will be its neighbor and how many common channels they share. Then the next step is to pick the maximum number of disjoint links (a maximum matching) and record the common available channels of each link.

The last step is to design a joint channel assignment and link scheduling algorithm such that the maximum number of links could be scheduled simultaneously. An intuitive idea is to maximize the channel utilization and let adjacent links use different channels so that they will not interfere with each other.

\section{Computing the Connectivity in CRAHNs}

As mentioned in Section IV, in our previous work on the complexity of the connectivity problem, we did not give a distributed algorithm for achieving the network connectivity. In this section, we discuss how to compute the connectivity in cognitive radio ad-hoc networks (CRAHNs).

\section{A. Problem Definition}

By using the same system and communication models as the capacity maximization problem in Section V-A and Section V-B, the connectivity computing problem is to construct a spanning tree over the secondary networks within the minimum number of timeslots.

\section{B. Related Work}

The connectivity problem has been extensively studied in conventional single-channel wireless ad-hoc networks under the SINR model [17], [18], [21], [30], [36]. This problem was first initiated in [36] where the authors gave a centralized algorithm which can construct the spanning tree in $O\left(\log ^{4} n\right)$ timeslots by using a clever non-linear power assignment. By first constructing a minimum spanning tree, the authors in [18] show that the minimum spanning tree can be scheduled in $O(\log n)$ timeslots under the SINR model. A variation of the connectivity problem is the "minimum-latency aggregation scheduling" problem which also requires building a spanning tree rooted at the sink node and scheduling the links in the tree within the minimum number of timeslots. The only difference is that, in aggregation, there is an additional requirement that links must be scheduled after all links below them in the tree are scheduled. For this minimum-latency aggregation scheduling problem, the authors in [30] gave a distributed algorithm with $O(\log K)$ timeslots where $K$ is the ratio between the longest link's length and the shortest link's length.

To our best knowledge, there are no distributed approximation algorithms for building a spanning tree over secondary networks under the SINR model. In paper [7], by using the SINR model, the authors aim to perform data collection in secondary networks as fast as possible. However, they only assume a single channel in the network, which makes the proposed algorithm inapplicable in cognitive radio ad-hoc networks where each node may have a different set of available channels.

\section{Preliminary Framework}

First of all, from the problem definitions, we can see that the connectivity computing problem is closely related to the capacity maximization problem. As discussed in Section V-E, by performing an asynchronous neighbor discovery algorithm, each secondary node will know who will be its neighbor and how many common available channels they share with each other. In this problem, we assume the secondary network topology is connected since otherwise there would not exist a spanning tree. There are many ways to find this tree in the connected secondary network. Finally, with this spanning tree, different from the capacity maximization problem, our focus is how to design a joint channel assignment and link scheduling algorithm that can schedule all the links in this spanning tree with the minimum number of timeslots. A straightforward strategy is to iteratively apply the designed distributed approximation algorithm for the capacity maximization problem until there are no links left.

\section{CONCLUSION}

Operating in cognitive radio ad-hoc networks (CRAHNs) is fundamentally different from conventional single-channel or multi-channel wireless ad-hoc networks. The dynamics of local available channels bring unique challenges to the design of CRAHNs, even when considering only the most basic operations such as message delivery, neighbor discovery and broadcasting. In this paper, we study two fundamental problems in CRAHNs, the capacity maximization problem and the connectivity problem. We summarize our recent results on these two problems, and discuss new extensions of the two problems. For capacity maximization, we propose combining channel assignment and scheduling to study distributed approximation algorithms under the physical interference model. For the connectivity problem, we propose to investigate distributed algorithms to achieve network connectivity within the minimum number of timeslots. We hope this work can inspire 
further study on these topics, especially on designing efficient distributed approximation algorithms.

\section{ACKNOWLEDGEMENTS}

This work was supported in part by the National Basic Research Program of China Grant 2011CBA00300, 2011CBA00302, the National Natural Science Foundation of China Grant 61103186, 61073174, 61033001, 61061130540, the Hi-Tech research and Development Program of China Grant 2006AA10Z216, and Hong Kong RGC-GRF grants $714009 \mathrm{E}$ and 714311.

\section{REFERENCES}

[1] I.F. Akyildiz, W.-Y. Lee, K.R. Chowdhury. CRAHNs: Cognitive radio ad-hoc networks. Ad Hoc Networks 7(5): 810-836, 2009.

[2] C.J.L. Arachchige, S. Venkatesan, R. Chandrasekaran, N. Mittal. Minimal Time Broadcasting in Cognitive Radio Networks. In ICDCN, 2011.

[3] C.J.L. Arachchige, S. Venkatesan, N. Mittal. An asynchronous neighbor discovery algorithm for cognitive radio networks. In DySPAN, 2008.

[4] C.J.L. Arachchige. Algorithms for neighbor discovery and broadcasting in cognitive radio networks. $\mathrm{PhD}$ Thesis, The University of Texas at Dallas, 2012.

[5] Y. Azar, O. Gurel-Gurevich, E. Lubetzky, T. Moscibroda. Optimal Discovery Strategies in White Space Networks. In ESA, 2011.

[6] P. Bahl, R. Chandra, T. Moscibroda, R. Murty, M. Welsh. White space networking with wi-fi like connectivity. In SIGCOMM, 2009.

[7] Z. Cai, S. Ji, J. He, A. G. Bourgeois. Optimal distributed data collection for asynchronous cognitive radio networks. In ICDCS, 2012.

[8] L. Cao, L. Yang, X. Zhou, Z. Zhang, H. Zheng. Optimus: SINR-driven spectrum distribution via constraint transformation. In DySPAN, 2010.

[9] L. Ding, T. Melodia, S.N. Batalama, J.D. Matyjas. Distributed routing, relay selection, and spectrum allocation in cognitive and cooperative adhoc networks. In SECON, 2010.

[10] L. Ding, T. Melodia, S.N. Batalama, M.J. Medley. ROSA: Distributed Joint Routing and Dynamic Spectrum Allocation in Cognitive Radio Ad Hoc Networks. In MSWiM, 2009.

[11] J. Dams, M. Hoefer, T. Kesselheim. Scheduling in wireless networks with rayleigh-fading interference. In SPAA, 2012

[12] B. Ellingsaeter. Binary spectrum allocation under SINR requirements. In DySPAN, 2012.

[13] Z. Feng, Y. Yang. Joint transport, routing and spectrum sharing optimization for wireless networks with frequency-agile radios. In INFOCOM, 2009.

[14] R. Gandhi, C.-C. Wang, Y.C. Hu. Fast rendezvous for multiple clients for cognitive radios using coordinated channel hopping. In SECON, 2012.

[15] O. Goussevskaia, M. M. Halldorsson, R. Wattenhofer. Algorithms for Wireless Capacity, CoRR abs/1203.0536, 2012.

[16] M. M. Halldorsson, P. Mitra. Wireless capacity and admission control in cognitive radio. In INFOCOM, 2012.

[17] M.M. Halldorsson, P. Mitra. Distributed connectivity of wireless networks. In PODC, 2012.

[18] M.M. Halldorsson, P. Mitra. Wireless connectivity and capacity. In SODA, 2012

[19] N. Hobbs, Y. Wang, Q.-S. Hua, D. Yu, F. C. M. Lau. Deterministic Distributed Data Aggregation under the SINR Model. In TAMC, 2012.

[20] F. Hou, J. Huang. Dynamic Channel Selection in Cognitive Radio Network with Channel Heterogeneity. In GLOBECOM, 2010.

[21] Q.-S. Hua, F. C. M. Lau. The scheduling and energy complexity of strong connectivity in ultra-wideband networks. In MSWiM, 2006.

[22] P. Hui, A. Chaintreau, J. Scott, R. Gass, J. Growcroft, C. Diot. Pocket switched networks and human mobility in conference environments. In SIGCOMM05 Workshops, 2005.

[23] J.J. Jaramillo, R. Srikant. Darwin: Distributed and adaptive reputation mechanism for wireless ad-hoc networks. In MOBICOM, 2007.

[24] T. Kesselheim. A Constant-Factor Approximation for Wireless Capacity Maximization with Power Control in the SINR Model. In SODA, 2011.

[25] Y. Kondareddy and P. Agrawal. Selective broadcasting in multi-hop cognitive radio networks. In IEEE Sarnoff Symposium, 2008.
[26] S. Krishnamurthy, M. Thoppian, S. Kuppa, R. Chandrasekaran, N. Mittal, S. Venkatesan, R. Prakash. Time-efficient distributed layer-2 autoconfiguration for cognitive radio networks. Computer Networks 52:831849, 2008.

[27] L.B. Le, E. Modiano, N. B. Shroff. Optimal control of wireless networks with finite buffers. In INFOCOM, 2010.

[28] M. Motani, V. Srinivasan, P. Nuggehalli. Peoplenet: Engineering a wirelessvirtual social network. In MOBICOM, 2005.

[29] H. Li, W. Huang, C. Wu, Z. Li, F. C. M. Lau. Utility-Maximizing Data Dissemination in Socially Selfish Cognitive Radio Networks. In MASS, 2011

[30] H. Li, Q.-S. Hua, C. Wu, F. C. M. Lau. Minimum-latency aggregation scheduling in wireless sensor networks under physical interference model. In MSWiM, 2010

[31] Q. Li, S. Zhu, G. Cao. Routing in Socially Selfish Delay Tolerant Networks. In INFOCOM, 2010.

[32] H. Liang, T. Lou, H. Tan, Y. Wang, D. Yu. Complexity of connectivity in cognitive radio networks through spectrum assignment. In ALGOSENSORS, 2012.

[33] H. Liu, Z. Lin, X. Chu, Y.-W. Leung. Taxonomy and Challenges of Rendezvous Algorithms in Cognitive Radio Networks. In ICNC, 2012.

[34] H. Liu, Z. Lin, X.-W. Chu, Y.-W. Leung. Jump-Stay Rendezvous Algorithm for Cognitive Radio Networks. IEEE Trans. on Parallel and Distrib. Syst., 23(10): 1867-1881, 2012.

[35] D. Lu, X. Huang, P. Li, J. Fan. Connectivity of large-scale cognitive radio ad-hoc networks. In INFOCOM, 2012.

[36] T. Moscibroda, R. Wattenhofer. The Complexity of Connectivity in Wireless Networks. In INFOCOM, 2006.

[37] M. J. Neely. Stochastic Network Optimization with Application to Communication and Queueing Systems. Morgan\&Claypool Publishers, 2010.

[38] M. J. Neely. Opportunistic Scheduling with Worst Case Delay Guarantees in Single and Multi-Hop Networks. In INFOCOM, 2011.

[39] W. Ren, Q. Zhao, A. Swami. Connectivity of cognitive radio networks: Proximity vs. opportunity. In CoRoNet, 2009.

[40] W. Ren, Q. Zhao, A. Swami. Power control in cognitive radio networks: How to cross a multi-lane highway. IEEE Journal on Selected Areas in Communications, 27(7):1283-1296, 2009.

[41] Y. Song, J. Xie. A distributed broadcast protocol in multi-hop cognitive radio ad-hoc networks without a common control channel. In INFOCOM, 2012.

[42] S. Srinivasa, S. Ali Jafar. The throughput potential of cognitive radio: a theoretical perspective. IEEE Communications Magazine, May 2007:73$79,2007$.

[43] J. Tang, S. Misra, G. Xue. Joint spectrum allocation and scheduling for fair spectrum sharing in cognitive radio wireless networks. Computer Networks 52(2008):2148-2158, 2008.

[44] L. Tassiulas, A. Ephremides. Stability properties of constrained queueing systems and scheduling policies for maximum throughput in multihop radio networks. IEEE Trans. Autom. Control, 37:1936-1948, 1992.

[45] N.C. Theis, R.W. Thomas, L.A. DaSilva. Rendezvous for cognitive radios. IEEE Trans. on Mobi. Comput., 10(2):216-227,2011.

[46] V. J. Venkataramanan, X. Lin, L. Ying, S. Shakkottai. On scheduling for minimizing end-to-end buffer usage over multihop wireless networks. In INFOCOM, 2010.

[47] P.-J. Wan, X. Jia, F. Yao. Maximum Independent Set of Links under Physical Interference Model. In WASA, 2009.

[48] B. Wang, K. J. R. Liu. Advances in cognitive radio networks: A survey. IEEE Journal of Selected Topics in Signal Processing, 5(1):5-23, 2011.

[49] B. Wang, Y. Wu, K. J. R. Liu. Game theory for cognitive radio networks: An overview. Computer Networks, 54:2537-2561, 2010.

[50] P. Wang, I. F. Akyildiz, A. M. Al-Dhelaan. Percolation Theory based Connectivity and Latency Analysis of Cognitive Radio Ad Hoc Networks. Wireless Networks, 17:659-669, 2011.

[51] D. Xue, E. Ekici. Guaranteed opportunistic scheduling in multi-hop cognitive radio networks. In INFOCOM, 2011.

[52] D. Yu, Q.-S. Hua, Y. Wang, F. C. M. Lau. An O(log n) Distributed Approximation Algorithm for Local Broadcasting in Unstructured Wireless Networks. In DCOSS, 2012.

[53] D. Yu, Q.-S. Hua, Y. Wang, H. Tan, F. C. M. Lau. Distributed MultipleMessage Broadcast in Wireless Ad-Hoc Networks under the SINR Model. In SIROCCO, 2012

[54] D. Yu, Y. Wang, Q.-S. Hua, F. C. M. Lau. Distributed $(\Delta+1)$-Coloring in the Physical Model. In ALGOSENSORS, 2011. 
[55] D. Yu, Y. Wang, Q.-S. Hua, F. C. M. Lau. Distributed local broadcasting algorithms in the physical interference model. In DCOSS, 2011.

[56] L. Yang, L. Cao, H. Zheng. Physical interference driven dynamic spectrum management. In DySPAN, 2008.

[57] S. Zhong, J. Chen, Y.R. Yang. Sprite: A simple, cheat-proof,credit-based system for mobile ad-hoc networks. In INFOCOM, 2003. 